\title{
USO DO CATETER CENTRAL DE INSERÇÃO PERIFÉRICA EM PACIENTES ADULTOS: UMA PERSPECTIVA PARA A ENFERMAGEM ONCOLÓGICA
}

\section{USE OF THE PERIPHERALLY INSERTED CENTRAL CATHETER IN ADULT PATIENTS: A PERSPECTIVE FOR ONCOLOGY NURSING}

\section{USO DEL CATÉTER CENTRAL DE INSERCIÓN PERIFÉRICA EN PACIENTES ADULTOS: UNA PERSPECTIVA PARA LA ENFERMERÍA ONCOLÓGICA}

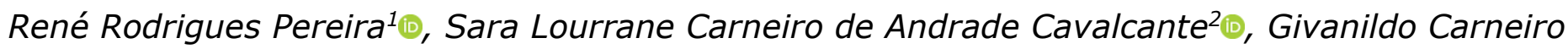
Benício $^{3}$, Aurora Pinheiro do Vale 4 , Dayane dos Reis Araújo Rocha ${ }^{5}$

\section{RESUMO}

Objetivo: Identificar a perspectiva para a enfermagem oncológica da utilização do PICC em pacientes adultos. Método: Revisão integrativa estruturada por estudos indexados nas bases de dados LILACS, MEDLINE e SciELO, incluindo artigos publicados entre os anos de 2015 e 2019, que abrangessem a experiência do PICC em pacientes adultos oncológicos. Resultados: Identificou-se inicialmente um total de 140 publicações, das quais nove compuseram a amostra final. 0 conteúdo permitiu a estruturação de quatro subtemas: comparação com outros dispositivos de acesso vascular; percepções de médicos e enfermeiros sobre o acesso venoso; experiência do paciente; e eventos adversos relacionados ao cateter. Assim, epilogou-se o aproveitamento de PICCs para o tratamento anticâncer, obtendo uma imagem clara do cenário de sua utilização na assistência de enfermagem oncológica. Conclusão: O PICC cresceu no escopo da enfermagem oncológica como tecnologia para acesso vascular. Entretanto, a perenidade do seu sucesso está inegavelmente vinculada à classificação adequada do cliente quanto à vulnerabilidade em desenvolver complicações e aos cuidados de enfermagem pós-inserção. A depender da preferência pessoal de oncologistas e usuários, o cenário futuro do PICC no tratamento de pacientes adultos com câncer é incerto.

Descritores: Neoplasias. Oncologia. Cateterismo Venoso Periférico. Cateterismo Venoso Central. Enfermagem Oncológica. Cuidados de Enfermagem.

\section{ABSTRACT}

Objective: To identify the perspective for oncology nursing of the PICC use in adult patients. Method: Integrative review structured by studies indexed in LILACS, MEDLINE, and SciELO databases, including articles published between the years 2015 and 2019 covering the experience of the PICC in adult oncology patients. Results: Initially, a total of 140 publications were identified, nine of which 
comprised the final sample. The content allowed the structuring of four subthemes: comparison with other vascular access devices; physicians' and nurses' perceptions of venous access; patient experience; and catheter-related adverse events. Thus, the use of PICCs for anticancer treatment was compiled, obtaining a clear picture of the scenario of their use in oncology nursing care. Conclusion: The PICC has grown in the scope of oncology nursing as a technology for vascular access. However, the longevity of its success is undeniably linked to the appropriate classification of the client as to vulnerability to develop complications and to the post-insertion nursing care. Depending on the personal preference of oncologists and users, the future scenario of the PICC in the treatment of adult cancer patients is uncertain.

Descriptors: Neoplasms. Oncology. Peripheral Venous Catheterization. Central Venous Catheterization. Oncologic Nursing. Nursing Care.

\section{RESUMEN}

Objetivo: Identificar la perspectiva para la enfermería oncológica del uso de PICC en pacientes adultos. Método: Revisión integradora estructurada por estudios indexados en las bases de datos LILACS, MEDLINE y SciELO, incluyendo artículos publicados entre los años 2015 y 2019, que abarquen la experiencia de PICC en pacientes oncológicos adultos. Resultados: Se identificó inicialmente un total de 140 publicaciones, de las cuales nueve conformaron la muestra final. El contenido permitió estructurar cuatro subtemas: comparación con otros dispositivos de acceso vascular; percepciones de médicos y enfermeros sobre el acceso venoso; experiencia del paciente; y eventos adversos relacionados con el catéter. Así, se epilogó el uso de los PICC para el tratamiento del cáncer, obteniendo un panorama claro del escenario de su uso en los cuidados de enfermería oncológica. Conclusión: El PICC ha crecido en el ámbito de la enfermería oncológica como tecnología para el acceso vascular. Sin embargo, la continuidad de su éxito está innegablemente ligada a la adecuada clasificación del cliente en cuanto a la vulnerabilidad en el desarrollo de complicaciones y a los cuidados de enfermería tras la inserción. Dependiendo de las preferencias personales de los oncólogos y de los usuarios, el escenario futuro del PICC en el tratamiento de pacientes adultos con cáncer es incierto.

Descriptores: Neoplasias. Oncología. Cateterismo Venoso Periférico. Cateterismo Venoso Central. Enfermería Oncológica. Cuidados de Enfermería.

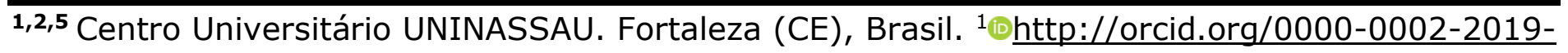
3263, 2ㅁttp://orcid.org/0000-0001-5827-0482, 5ำttp://orcid.org/0000-0002-1902-5019

${ }^{3}$ Universidade Federal do Ceará/UFCE. Fortaleza (CE), Brasil. ${ }^{3}$ ịttp://orcid.org/0000-0001-9642- 
4 Universidade Estadual do Ceará/UECE. Fortaleza (CE), Brasil. ${ }^{4}$ http://orcid.org/0000-0001$\underline{6246-4829}$

\begin{abstract}
Como citar este artigo
Pereira RR, Cavalcante SLCA, Benício GC, Vale AP, Rocha DRA. Uso do Cateter Central de Inserção Periférica em Pacientes Adultos: Uma Perspectiva para a Enfermagem Oncológica. Rev enferm UFPE on line. 2021;15:e277934 DOI: https: / / doi.org/10.5205/1981-8963.2021.247934
\end{abstract}

\section{INTRODUÇÃO}

O cateter venoso central de inserção periférica (PICC, como é reconhecido internacionalmente por sua abreviatura na língua inglesa) configura-se como um dispositivo com grande aplicabilidade no cuidado de pacientes que necessitam de um acesso à circulação central ${ }^{1,2}$. Ao longo de quase um século no escopo do cateterismo, os PICCs, entendidos hoje como cateteres venosos centrais em material biocompatível inseridos por orientação ultrassonográfica em veias profundas do braço, foram introduzidos no mercado em meados dos anos 90 , e desde então têm sido objeto de estudo para a concessão de um serviço seguro e eficaz de assistência à saúde ${ }^{3}$.

O fundamental atributo desses acessos é que, apesar de geralmente apresentarem sítio de inserção localizado no terço médio de membros superiores, sua ponta emerge na junção cavoatrial, a saber, a confluência da veia cava superior com o átrio direito, conferindo às soluções perfundidas maior hemodiluição, sendo capazes, assim, de serem usados para qualquer tipo de infusão, independentemente do $\mathrm{pH}$, da osmolaridade ou do potencial efeito prejudicial ao endotélio, bem como para a monitorização hemodinâmica ${ }^{4}$.

Ademais, de acordo com Pittiruti e Scoppettuolo ${ }^{5}$, que consideram adequado o dispositivo cujos benefícios à saúde excedem as consequências negativas esperadas em pacientes clínicos em geral, esses cateteres demonstram exequibilidade de aplicação logo a partir do sexto dia de duração proposta para a Terapia Intravascular (TIV). Além disso, mesmo havendo adequação de outros dispositivos, períodos superiores a 31 dias, por exemplo, não o inviabilizam; ao contrário, atenta-se que mecanismos mais invasivos devem ser reservados para cenários onde o PICC não seja praticável, como calibre de veias inadequado ou infusões episódicas ao longo de vários meses.

Diante de oportunas particularidades, esse cateter rapidamente tornou-se estimado para a terapêutica de um largo espectro de pacientes oncológicos ${ }^{6,7}$. Sendo o profissional de enfermagem, além de legalmente apto à sua inserção ${ }^{8}$, o componente da equipe que mais permanece em contato com esse público no manejo dos cuidados factíveis à prática resolutiva e qualificada, torna-se incontestável o seu profundo envolvimento com uma tecnologia tão emergente. Todavia, assim como acessos centrais tradicionais, a utilização do PICC associa-se, ainda, a uma considerável incidência de Eventos Adversos (EAs), com destaque às complicações infecciosas e trombóticas ${ }^{9,10}$. 
Em teoria legítima na contemporaneidade, Virchow (1821-1902) foi o primeiro a descrever os mecanismos de desenvolvimento do câncer e trombose. Sua tríade delineia três grandes elementos causais que exercem influência acerca da fisiopatologia da Trombose Venosa Profunda (TVP), sinalizando alterações no fluxo sanguíneo, injúria vascular, e hipercoagulabilidade como princípios desencadeantes do processo de ativação dos fatores de coagulação, cujo desfecho resulta na formação do trombo ${ }^{11}$.

Além disso, o risco de infecção causada por PICCs relaciona-se profundamente ao tempo de permanência do cateter e aos cuidados aplicados por parte da equipe de profissionais responsáveis pelo seu manuseio, apontando, além da necessidade de um cuidado especializado, a relevância da familiaridade com as recomendações científicas mais recentes sobre o tema ${ }^{12,13}$.

Não seria notoriamente inacreditável a aproximação de complicações trombóticas a pacientes oncológicos portadores de um cateter que pode alcançar o comprimento de $60 \mathrm{~cm}$, cujas comorbidades e condições clínicas, acrescidas dos próprios aspectos relacionados ao tumor, desestabilizam a homeostasia hemodinâmica ${ }^{9,14,15}$. Não esperaríamos tampouco que uma ferramenta destinada à TIV prolongada, implantada em clientela imunocomprometida, susceptível à manipulação constante de profissionais com o mais heterogêneo rigor de treinamento, estivesse isenta de qualquer episódio de contaminação. Nesse sentido, a concordância desastrosa de todos esses elementos em um único grupo de pacientes favoreceria consequências catastróficas.

Mesmo diante de famigerados EAs, na exiguidade de evidências científicas suficientes, diretrizes nacionais e internacionais curiosamente limitam-se à exigência de cateteres de linha central para a perfusão de medicamentos irritantes e/ou vesicantes, embora não preconizem um dispositivo específico para a administração de quimioterapia ${ }^{4,16}$, facultando a escolha pelo tipo de acesso a uma tomada compartilhada de decisão entre oncologistas, enfermeiros e pacientes ${ }^{17}$.

É nesse ínterim, permeado por benefícios e complicações, e por conta da rapidez do desenvolvimento tecnológico e transitoriedade das indicações clínicas, que se ressalta a necessidade de uma compilação das evidências científicas sobre o aproveitamento de PICCs para a TIV do tratamento anticâncer, regulando-se uma pesquisa com a seguinte questão norteadora: Qual a perspectiva, para a enfermagem oncológica, de utilização do PICC em pacientes adultos?

A promoção de um melhor entendimento sobre o compromisso de pesquisadores fundamentados na entrega de uma assistência à saúde de qualidade, bem como sobre os progressos empreendidos com o intuito de mitigar efeitos adversos à terapêutica orientarão esses profissionais em suas condutas de rotina, oferecendo acesso à informação de maneira rápida e concisa. 
À vista disso, sobretudo ponderando a respeito da segurança e bem-estar do público dessa especialidade clínica, esta pesquisa de revisão objetiva identificar a perspectiva para a enfermagem oncológica no que se refere à utilização do PICC em pacientes adultos.

\section{OBJETIVO}

Identificar a perspectiva para a enfermagem oncológica no que se refere à utilização do PICC em pacientes adultos.

\section{MÉTODO}

\section{Desenho da pesquisa}

Para a identificação das produções, optou-se pela elaboração de uma revisão integrativa (RI), tendo como um dos seus objetivos proporcionar aos profissionais da área da saúde, submetidos a longas jornadas de trabalho e caracterizados pelo tempo restrito, a disseminação eficaz da informação ${ }^{18}$. Esse método conserva-se como um instrumento importante da Prática Baseada em Evidências (PBE), assinalando uma abordagem voltada ao cuidado e ao ensino firmado em pesquisas preexistentes, conduzidas dentro dos preceitos científicos, o que de fato entende-se como evidência ${ }^{19}$.

Diante da divergência entre muitos autores sobre sua definição e sistematização ${ }^{20}$, esta pesquisa considera RI como uma abordagem ampla, que pondera estudos experimentais e não experimentais para a compreensão completa de uma temática específica, determinando seu conceito atual através da análise de teorias e evidências que contribuam para uma discussão proveitosa sobre a qualidade do cuidado prestado ao paciente, tendo o potencial de desempenhar um papel importante na PBE da enfermagem ${ }^{21}$.

Em consonância com Souza, Silva e Carvalho ${ }^{19}$, o processo de elaboração orientou-se por seis etapas e seus respectivos complementos metodológicos: Elaboração da Pergunta Norteadora; Busca ou Amostragem na Literatura; Coleta de Dados; Análise Crítica dos Estudos Incluídos; Discussão dos Resultados; e Apresentação da Revisão Integrativa - pormenorizados a seguir.

\section{Elaboração da pergunta norteadora}

Visto como o momento mais significativo e com o intuito de desenvolver o mesmo rigor de qualquer outro método científico ${ }^{18}$, na primeira etapa desta revisão, para a construção da pergunta da pesquisa, empregou-se a estratégia PICO, proposta pela PBE para a decomposição e organização de impasses que surgem na prática assistencial, de ensino ou pesquisa ${ }^{22}$.

De acordo com Santos, Pimenta e Nobre ${ }^{22}$, a estratégia PICO representa, no cenário clínico, um acrônimo para Paciente, Intervenção, Comparação e Outcomes (desfecho), proporcionando maior acurácia da informação disponível e reduzindo tempo dispendioso com buscas desnecessárias. Em pesquisas não clínicas, entretanto, o "I" funciona como abreviatura para Interesse, ao passo que as 
letras "C" e "O" se reúnem na representação da palavra Contexto. Nessa perspectiva, todos os componentes relacionados ao problema seguem descritos no quadro 1.

Quadro 1. Apresentação da estratégia PICO para a identificação dos descritores controlados

\begin{tabular}{|l|l|}
\hline $\mathbf{P}$ & $\begin{array}{l}\text { Pacientes adultos diagnosticados com qualquer tipo de câncer, incluindo neoplasias oncológicas e } \\
\text { hematológicas em atendimento hospitalar ou domiciliar. }\end{array}$ \\
\hline I & $\begin{array}{l}\text { Dispositivos centrais de inserção periférica. Sem limitação quanto à empresa de fabricação, } \\
\text { quantidade de lúmens, presença de válvula, calibre, tamanho, profissional responsável pelo } \\
\text { procedimento de inserção, sítio de localização da punção ou curativo de proteção. }\end{array}$ \\
\hline Co & $\begin{array}{l}\text { Enfermagem oncológica no tratamento antineoplásico de médio a longo prazo, cujas características } \\
\text { das soluções sejam incompatíveis à perfusão por via periférica. }\end{array}$ \\
\hline
\end{tabular}

Fonte: Elaboração própria.

Diante do cerne categoricamente constituído, estruturou-se a seguinte pergunta norteadora: Qual a perspectiva, para a enfermagem oncológica, de utilização do PICC em pacientes adultos?

\section{Busca ou amostragem na literatura}

A fim de facilitar o processo de investigação, a determinação dos termos de busca seguiu consulta aos Descritores em Ciências da Saúde (DeCS) utilizados para a localização dos artigos, baseando-se nos elementos constituintes da estratégia em uso na etapa anterior. Ressalta-se que, em território de pesquisa internacional, seus equivalentes em inglês assumiram o espaço nas ferramentas de busca. Assim, consideraram-se seis descritores controlados: Neoplasias (Neoplasms), Oncologia (Oncology), Cateterismo Venoso Periférico (Catheterization, Peripheral), Cateterismo Venoso Central (Catheterization, Central Venous), Enfermagem Oncológica (Oncology Nursing), e Cuidados de Enfermagem (Nursing Care).

Para garantir a representatividade da amostra e visando à amplitude dos achados, realizou-se um trabalho abrangente na literatura eletrônica durante os meses de fevereiro e março de 2020, que consistiu numa pesquisa de estudos indexados nas bases de dados Literatura Latino-Americana e do Caribe em Ciências da Saúde (LILACS), Medical Literature Analysis Retrieval System Online (MEDLINE) e Scientific Electronic Library Online (SciELO).

Fez-se nesse momento o primeiro tratamento dos achados, incluindo unicamente artigos cujas publicações contemplassem o recorte temporal compreendido entre os anos de 2015 e 2019, bem como aqueles disponíveis gratuitamente em texto completo. Pela facilidade de ferramentas de tradução online, o idioma não impôs entraves à compreensão do conteúdo. Assim, todos foram considerados adequados para a composição da amostra. Desse modo, com o cruzamento dos descritores previamente identificados e em uso da lógica booleana, identificou-se inicialmente um total de 140 publicações, das quais 131 foram referências obtidas na base de dados MEDLINE, 04 na LILACS, e 05 na SCIELO.

Diante do material coletado, 37 publicações foram excluídas por apresentarem-se em duplicidade face ao resultado das correspondentes combinações nas diferentes bases de dados, totalizando 103 
artigos para análise posterior. Seguiu-se, então, com a leitura criteriosa dos títulos e resumos, etapa que excluiu duas abordagens científicas de revisão, 28 estudos por discorrerem unicamente sobre outros dispositivos de acesso vascular central, 13 por tratarem de pacientes oncológicos pediátricos, e 32 por não contemplarem a temática pretendida. Por fim, sobre as restantes 28 produções, aquelas que indicaram resposta à pergunta norteadora compuseram uma amostra final de nove artigos (Quadro 2).

Salienta-se que, nessas considerações para a composição da amostra, algumas produções apresentaram adequabilidade para mais de um fator de exclusão. Dessa forma, os itens foram excluídos e contabilizados de acordo com a ordem supracitada dos critérios aplicados. Ainda, nessa busca, nenhum estudo de revisão abordando exclusivamente a experiência do PICC em pacientes adultos durante tratamento anticâncer foi identificado, salvaguardando a originalidade da pesquisa.

Quadro 2. Fluxograma Prisma

\begin{tabular}{|c|c|c|}
\hline \multirow[t]{3}{*}{ Identificação } & \multirow{3}{*}{$\begin{array}{l}\text { Resultados da pesquisa } \\
\qquad(\mathrm{n}=140)\end{array}$} & MEDLINE $(n=131)$ \\
\hline & & LILACS $(n=04)$ \\
\hline & & SciELO $(n=05)$ \\
\hline \multirow[t]{2}{*}{ Seleção } & $\begin{array}{c}\text { Total } \\
(\mathrm{n}=140)\end{array}$ & $\begin{array}{l}\text { Publicações excluídas } \\
\text { por serem duplicatas } \\
\qquad(\mathrm{n}=37)\end{array}$ \\
\hline & $\begin{array}{c}\text { Total } \\
(\mathrm{n}=103)\end{array}$ & $\begin{array}{l}\text { Publicações excluídas } \\
\text { após leitura de títulos } \\
\text { e resumos }(n=75)\end{array}$ \\
\hline Elegibilidade & \multicolumn{2}{|c|}{$\begin{array}{l}\text { Artigos com análise completa de conteúdo } \\
\qquad(\mathrm{n}=28)\end{array}$} \\
\hline Incluídos & \multicolumn{2}{|c|}{$\begin{array}{l}\text { Total de estudos incluídos na amostra } \\
\qquad(\mathrm{n}=09)\end{array}$} \\
\hline
\end{tabular}

Fonte: Galvão, Pansani, Andrade ${ }^{23}$.

\section{Coleta de dados}

Para condensar as informações relevantes contidas nas fontes deliberadas, optou-se pelo Instrumento de Ursi, adaptado. Conforme Ursi ${ }^{24}$, além de proporcionar ao revisor o acesso à informação de maneira prática e compilada, a existência de um questionário previamente elaborado permite avaliar a individualidade de cada artigo, determinar os métodos empregados e o subsídio que proporciona ao problema da pesquisa em questão, mitigando o risco de erros na transcrição dos resultados e fomentando veracidade e confiabilidade diante da análise crítica do leitor.

Assim, na extração dos dados de identificação do artigo, utilizaram-se as seguintes informações: título, autores, ano de publicação, designação do periódico, idioma e país de publicação (Quadro 3). Por sua vez, as características metodológicas dos estudos compuseram outra sessão, contendo os seguintes itens: método, nível de evidência, objetivos, e conclusões da investigação (Quadro 4). 
Análise crítica dos estudos incluídos

A partir da abordagem metodológica adotada, considerou-se um sistema hierárquico de evidências composto por seis níveis ${ }^{19}$ :

Nível 1: evidências resultantes da meta-análise de múltiplos estudos clínicos controlados e randomizados.

Nível 2: evidências obtidas em estudos individuais com delineamento experimental.

Nível 3: evidências de estudos quase-experimentais.

Nível 4: evidências de estudos descritivos (não-experimentais) ou com abordagem qualitativa.

Nível 5: evidências provenientes de relatos de caso ou de experiência.

Nível 6: evidências baseadas em opiniões de especialistas.

\section{RESULTADOS}

Nove artigos preencheram os critérios de inclusão relatando aspectos da prática clínica e/ou experiência do paciente em relação ao uso do PICC. Com 44,4\% (n = 4) das publicações, a China apresentou incontestável preponderância sobre outras nacionalidades (estudos 1, 2, 3 e 8), enquanto o restante da amostra foi composto por 22,2\% $(n=2)$ de estudos originários da Itália (estudos 4 e 9) e 33,3\% ( $n=3$ ) equitativamente distribuído entre Canadá (estudo 7), Espanha (estudo 6) e Estados Unidos (estudo 5). À exceção de um único estudo publicado em chinês (estudo 1), todos os outros estavam disponíveis em inglês.

Quadro 3. Identificação dos estudos constituintes da amostra

\begin{tabular}{|c|c|c|c|c|}
\hline Ordem & Título & $\begin{array}{l}\text { Autores e ano } \\
\text { de publicação }\end{array}$ & Periódico & $\begin{array}{l}\text { País e } \\
\text { idioma }\end{array}$ \\
\hline 1. & $\begin{array}{l}\text { Retrospective Analysis of Peripherally Inserted Central } \\
\text { Catheter-related Vein Thrombosis in Lung Cancer } \\
\text { Patients }\end{array}$ & $\begin{array}{l}\text { CHEN; YU; LI, } \\
2015\end{array}$ & $\begin{array}{l}\text { Chinese } \\
\text { Journal of } \\
\text { Lung Cancer }\end{array}$ & $\begin{array}{l}\text { China, } \\
\text { chinês }\end{array}$ \\
\hline 2. & $\begin{array}{l}\text { High risk of deep vein thrombosis associated with } \\
\text { peripherally inserted central catheters in lymphoma. }\end{array}$ & ZANG et al., 2016 & Oncotarget & $\begin{array}{l}\text { China, } \\
\text { inglês }\end{array}$ \\
\hline 3. & $\begin{array}{l}\text { Comparison of three types of central venous catheters } \\
\text { in patients with malignant tumor receiving } \\
\text { chemotherapy. }\end{array}$ & FANG et al., 2017 & $\begin{array}{l}\text { Patient } \\
\text { preference } \\
\text { and } \\
\text { adherence }\end{array}$ & $\begin{array}{l}\text { China, } \\
\text { inglês }\end{array}$ \\
\hline 4. & $\begin{array}{l}\text { Clinical management of peripherally inserted central } \\
\text { catheters compared to conventional central venous } \\
\text { catheters in patients with hematological malignancies: } \\
\text { A large multicenter study of the REL GROUP. }\end{array}$ & $\begin{array}{l}\text { FRANCCHIOLA et } \\
\text { al., } 2017\end{array}$ & $\begin{array}{l}\text { American } \\
\text { Journal of } \\
\text { Hematology }\end{array}$ & $\begin{array}{l}\text { Itália, } \\
\text { inglês }\end{array}$ \\
\hline 5. & $\begin{array}{l}\text { Incidence of and factors associated with catheter- } \\
\text { related bloodstream infection in patients with } \\
\text { advanced solid tumors on home parenteral nutrition } \\
\text { managed using a standardized catheter care protocol. }\end{array}$ & VASHI et al., 2017 & $\begin{array}{l}\text { BMC } \\
\text { Infectious } \\
\text { Diseases }\end{array}$ & $\begin{array}{l}\text { Estados } \\
\text { Unidos, } \\
\text { inglês }\end{array}$ \\
\hline 6. & $\begin{array}{l}\text { Living with a peripherally inserted central catheter: the } \\
\text { perspective of cancer outpatients - a qualitative study. }\end{array}$ & $\begin{array}{l}\text { BRAVO et al., } \\
2018\end{array}$ & $\begin{array}{l}\text { Support Care } \\
\text { Cancer }\end{array}$ & $\begin{array}{l}\text { Espanha, } \\
\text { inglês }\end{array}$ \\
\hline
\end{tabular}


9. Can Peripherally Inserted Central Catheters Be Safely CAMPAGNA et al., The Itália, Placed in Patients with Cancer Receiving 2019 Oncologist inglês Chemotherapy? A Retrospective Study of Almost 400,000 Catheter-Days.

Fonte: Adaptado do Instrumento de Ursi ${ }^{24}$.

Dois estudos compararam a segurança e eficiência do PICC com outros dispositivos centrais utilizados para a quimioterapia (estudos 3 e 4). Dois descreveram a experiência do paciente em uso do cateter (estudos 3 e 6), enquanto a opinião de especialistas foi pautada apenas no artigo de Levasseur et al. ${ }^{16}$, sendo este, também, o único de nível 6 de evidência. Todas as outras publicações apresentaram nível 4. Os EA relacionados ao PICC, por sua vez, foram criteriosamente explorados em cinco das publicações (estudos 1, 2, 5, 8 e 9).

Quadro 4. Análise de conteúdo dos estudos constituintes da amostra

\begin{tabular}{|c|c|c|c|}
\hline Ordem & Método e evidência & Objetivo & Conclusão \\
\hline 1. & $\begin{array}{l}\text { Análise retrospectiva, } \\
\text { nível } 4\end{array}$ & $\begin{array}{l}\text { Analisar os fatores que } \\
\text { causam trombose venosa } \\
\text { relacionada ao PICC e } \\
\text { encontrar intervenções de } \\
\text { enfermagem apropriadas à } \\
\text { sua atenuação. }\end{array}$ & $\begin{array}{l}\text { A longevidade do aproveitamento do } \\
\text { cateter depende da implementação de } \\
\text { cuidados individuais de enfermagem que } \\
\text { envolvam a avaliação da condição do } \\
\text { paciente no que se refere ao sexo, veia } \\
\text { residente e valor de fibrinogênio, } \\
\text { diminuindo a ocorrência de trombose } \\
\text { venosa relacionada ao PICC. }\end{array}$ \\
\hline 2. & $\begin{array}{l}\text { Estudo retrospectivo, } \\
\text { nível } 4\end{array}$ & $\begin{array}{l}\text { Contrapor a incidência de } \\
\text { trombose associada ao PICC } \\
\text { no linfoma com outros tipos } \\
\text { de câncer. }\end{array}$ & $\begin{array}{l}\text { O uso do cateter pode ser minimizado em } \\
\text { pacientes com linfoma pela maior } \\
\text { predisposição desse público em } \\
\text { desenvolver trombose relacionada ao } \\
\text { PICC. }\end{array}$ \\
\hline 3. & $\begin{array}{l}\text { Estudo de } \\
\text { prospectivo, } \\
\text { nível } 4\end{array}$ & $\begin{array}{l}\text { Comparar as complicações, } \\
\text { o custo e a qualidade de } \\
\text { vida dos pacientes em uso } \\
\text { de três tipos de Cateteres } \\
\text { Venosos Centrais (CVC) } \\
\text { utilizados rara } \\
\text { quimioterapia. }\end{array}$ & $\begin{array}{l}\text { Dispositivos totalmente implantados } \\
\text { (port-a-caths) podem ser eleitos no lugar } \\
\text { do PICC por evidência de menos } \\
\text { complicações e maior qualidade de vida e } \\
\text { satisfação dos pacientes, podendo ser } \\
\text { estratégias utilizadas pela enfermagem. }\end{array}$ \\
\hline 4. & $\begin{array}{l}\text { Estudo observacional, } \\
\text { nível } 4\end{array}$ & $\begin{array}{l}\text { Comparar a incidência de } \\
\text { eventos infecciosos e } \\
\text { trombóticos em PICC e CVC } \\
\text { convencionais em pacientes } \\
\text { onco-hematológicos. }\end{array}$ & $\begin{array}{l}\text { O PICC demonstra expectativa positiva de } \\
\text { uso por apresentar-se como uma } \\
\text { alternativa segura e eficaz aos CVC, } \\
\text { exibindo uma vida útil mais longa e } \\
\text { sobrevida livre de EA relacionados ao } \\
\text { cateter. }\end{array}$ \\
\hline 5. & $\begin{array}{l}\text { Estudo retrospectivo, } \\
\text { nível } 4\end{array}$ & \begin{tabular}{llr|} 
Avaliar a & incidência e os \\
fatores & associados & à \\
Infecção & da & Corrente \\
Sanguínea & Relacionada & ao \\
Cateter & (ICSRC) & em \\
pacientes & com càncer \\
submetidos & à & Nutrição \\
Parenteral Total (NPT).
\end{tabular} & $\begin{array}{l}\text { Protocolos de assistência de enfermagem } \\
\text { padronizados de manutenção de cateter } \\
\text { predizem uma baixa taxa de ICSRC e } \\
\text { ampliam o uso do dispositivo, } \\
\text { evidenciando que sua incidência pode não } \\
\text { se relacionar ao tipo de acesso venoso, } \\
\text { mas à necessidade de cuidados } \\
\text { especializados pós-inserção. }\end{array}$ \\
\hline 6. & $\begin{array}{l}\text { Estudo qualitativo } \\
\text { fenomenológico, } \\
\text { nível } 4\end{array}$ & $\begin{array}{l}\text { Descrever a experiência de } \\
\text { uso do PICC em pacientes } \\
\text { com câncer. }\end{array}$ & $\begin{array}{l}\text { A maioria dos pacientes considerou ter } \\
\text { uma linha PICC como uma experiência } \\
\text { positiva que recomendaria a outros } \\
\text { pacientes, pois descobriu que isso não } \\
\text { alterava sua qualidade de vida. }\end{array}$ \\
\hline 7. & $\begin{array}{l}\text { Pesquisa de opinião de } \\
\text { especialistas, } \\
\text { nível } 6\end{array}$ & $\mid \begin{array}{lrr}\text { Avaliar as } & \text { práticas, } \\
\text { percepções } & \text { de } \\
\text { complicações e } & \text { risco do } \\
\text { acesso venoso } & \text { entre }\end{array}$ & 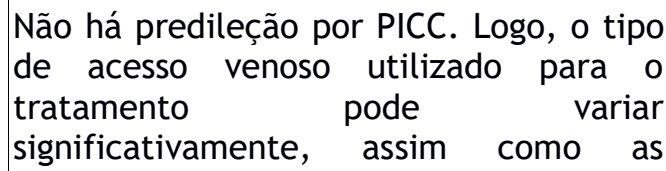 \\
\hline
\end{tabular}


enfermeiros e médicos percepções sobre os riscos oncologistas.

\begin{tabular}{|c|c|c|c|}
\hline 8. & $\begin{array}{l}\text { Análise retrospectiva, } \\
\text { nível } 4\end{array}$ & $\begin{array}{l}\text { Explorar os fatores que } \\
\text { potencializam o risco de } \\
\text { infecção causada por PICCs } \\
\text { em pacientes com tumor } \\
\text { ósseo. }\end{array}$ & $\begin{array}{l}\text { As infecções causadas por PICCs foram } \\
\text { relacionadas à experiência dos } \\
\text { profissionais responsáveis pela inserção e } \\
\text { ao tempo de permanência do cateter. }\end{array}$ \\
\hline 9. & $\begin{array}{l}\text { Estudo retrospectivo, } \\
\text { nível } 4\end{array}$ & \begin{tabular}{|lrr} 
Verificar & os & EAs \\
relacionados ao & PICC & em \\
pacientes com & neoplasias \\
oncológicas & & ou \\
hematológicas. & &
\end{tabular} & $\begin{array}{l}\text { Perspectiva otimista de uso do PICC para } \\
\text { a administração de quimioterapia, pois } \\
\text { associou-se a uma baixa taxa de todos os } \\
\text { EAs. }\end{array}$ \\
\hline
\end{tabular}

Fonte: Adaptado do Instrumento de Ursi ${ }^{24}$.

O levantamento do conteúdo permitiu a estruturação de quatro subtemas: comparação com outros dispositivos de acesso vascular; eventos adversos relacionados com o cateter; experiência do paciente; percepções de médicos e enfermeiros sobre o acesso venoso.

A incidência de episódios trombóticos e infecciosos foi relatada em 100\% dos estudos que incluíam a identificação de complicações relacionadas ao dispositivo de acesso central entre os seus objetivos. Outros inconvenientes descritos vincularam-se à flebite, oclusão do lúmen, ruptura, má posição da ponta e síndrome de pinch-off, definida como o cateter dobrado, comprimido ou mesmo fragmentado no espaço estreito entre a clavícula e a primeira costela (estudo 3).

Os artigos revisados envolveram a análise de 13.464 indivíduos. Um total de 13.382 pacientes oncológicos foram investigados no que concerne à frequência de EA relacionados ao cateter e/ou qualidade de vida, conforto e satisfação. Dois estudos não apresentaram distinção entre o sexo dos participantes ingressados no início da coleta de dados (estudos 4 e 8). Todavia, dos 12.541 pacientes restantes, $57,3 \%(n=7.194)$ eram homens e $42,6 \%(n=5.347)$, mulheres. Apenas uma publicação centrava-se na interpelação de oncologistas e enfermeiros oncológicos sobre as práticas de acesso, percepções de complicações e risco, apresentando, assim, uma outra clientela como objeto de estudo (estudo 7).

Além da evidente indicação para infusão intravenosa prolongada ou contínua de agentes quimioterápicos, o uso do PICC também foi conveniente para pacientes com acesso venoso limitado, coleta intermitente de amostras sanguíneas (estudo 6), Nutrição Parenteral Total - NPT (estudo 5) e estrutura vascular inapropriada à terapêutica desejada (estudo 2).

Em 66,6\% $(n=6)$ dos estudos, o enfermeiro, adequadamente especializado, foi referido como o profissional responsável pelo procedimento de inserção (estudos 7, 1, 5, 2, 3 e 6). Os PICCs utilizados variaram em conformações, apresentando diâmetros entre 4 e $6 \mathrm{Fr}$ (estudos 2, 3 e 9), 25 a $55 \mathrm{~cm}$ de comprimento (estudo 9), com extremidade distal valvulada ou aberta (estudos 2 e 9) e lúmen simples ou duplo (estudos 2, 3 e 4).

As veias basílica, cefálica, braquial e cubital mediana foram reportadas como de escolha à canulação em $44,4 \%(n=4)$ dos artigos (estudos $1,2,3$ e 5), havendo favoritismo da basílica em $50 \%(n=2)$ 
destes (estudos 2 e 3). Não houve especificidade quanto ao vaso periférico puncionado nos demais estudos.

\section{DISCUSSÃO}

\section{Comparação com outros dispositivos de acesso vascular}

$\mathrm{Na}$ inexistência de recomendação específica que determine o tipo de Dispositivo de Acesso Vascular (DAV) para os pacientes adultos durante o tratamento antineoplásico, sejam esses tumores sólidos ou hematológicos, o PICC configura-se como uma alternativa persistente no processo de escolha ${ }^{17,25}$. Essa categoria de cateter cujas vantagens encontram-se desde o momento de inserção, com a redução do ônus hospitalar em reservas de salas cirúrgicas, uma vez que o procedimento pode ser realizado à beira leito, garante a redução do extravasamento da quimioterapia, evita repetidas punções venosas ${ }^{5}$ e, apesar de apresentar-se continuamente envolto por controvérsias sobre sua segurança e eficácia, fornece acesso vascular conveniente para pacientes oncológicos ${ }^{13}$.

Em comparação direta de EA entre CVC convencionais e de inserção periférica, o estudo de Fracchiolla et al. ${ }^{2}$ exibe uma vida útil significativamente mais longa ao PICC, relacionando-o a uma menor taxa de episódios infecciosos e remoção devido a complicações, sem destaque estatístico, inclusive, ao desenvolvimento de trombos.

Corroborando esses achados, entre os 2.477 pacientes incluídos no período de 7 anos no estudo de Campagna et al. $^{3}$, apenas 419 EA foram relacionados ao PICC, conferindo um baixo índice de complicações durante a administração de quimioterapia. Os autores, porém, destacam a importância da implementação de protocolos de cuidados com o cateter e programas de educação para a equipe de enfermagem que lida diariamente com esses dispositivos, visto que episódios de oclusões e infecções podem estar associados diretamente à qualidade da assistência pós-inserção.

Outra prerrogativa desses cateteres largamente documentada na literatura internacional é a de que sua inserção, anteriormente competência exclusiva dos médicos, consiste em conduta legal às ações do profissional enfermeiro ${ }^{6,14}$, diminuindo o tempo de espera do procedimento e consequente início do tratamento ${ }^{17}$. No Brasil, entretanto, apesar da Resolução $n^{\circ}$ 258/2001 do COFEN, que versa sobre a legitimidade da atuação de enfermeiros habilitados nessa área, a prática ainda é evidenciada pela hegemonia médica em centros cirúrgicos ${ }^{26,27}$, atravancando o usufruto dos benefícios de implantação do PICC e mitigando a evidência clínica de sua utilização, haja vista a escassez de publicações científicas relacionadas.

Em proveito à pluralidade dos dispositivos, admite-se que cada uma das vias de administração endovenosa tem seus próprios méritos e complicações, precisando esses fatores serem pesados para qualquer público, a partir de uma abordagem individualizada. Para tanto, durante a tomada de decisão, que usualmente está vinculada à preferência pessoal do profissional oncologista ${ }^{17,28}$, sugere- 
se que alguns fatores sejam considerados, tais como: peculiaridades clínicas do paciente ${ }^{6,14}$, características das soluções de infusão, profissional disponível para a inserção ${ }^{16}$, duração do acesso ${ }^{13}$, e continuação da TIV em ambiente domiciliar ${ }^{12}$.

\section{Percepções de médicos e enfermeiros sobre o Acesso Venoso}

No estudo de LeVasseur et al. ${ }^{17}$, considerando isoladamente a opinião de médicos oncologistas e as características da infusão durante esquemas quimioterápicos compatíveis de perfusão por via periférica, PICCs são indicados somente após a preferência por acessos periféricos. Ademais, para regimes incompatíveis, pela liberdade do paciente de realizar atividades da vida diária sem limitação e pela natureza de longo prazo da terapêutica, esses profissionais indicam o dispositivo somente após a predileção por “ports”. Desta forma, a depender desta avaliação, o PICC em nenhuma situação configura-se como dispositivo de primeira escolha.

Apesar de concordarem com a classe médica sobre a escolha do dispositivo diante desses diferentes cenários, enfermeiros apontam vantagens importantes de uso do PICC relacionadas a menos tentativas de punções venosas em acessos problemáticos, proporcionando maior conforto e reduzindo a ansiedade dos pacientes.

\section{Experiência do paciente}

Sendo o usuário alvo direto dos benefícios e complicações associados à seleção do cateter, reconhecese a soberania do seu ponto de vista, bem como a importância de inseri-lo no plano terapêutico ${ }^{28}$, tornando-o sujeito ativo nesse processo e fornecendo subsídios conceituais importantes para sua prática do autocuidado, desenvolvendo, assim, um cenário adequado ao bom progresso do tratamento $^{12}$.

Nessa seara, o estudo realizado por Fang et al. ${ }^{25}$, que também comparou as complicações e custo de três DAV comumente usados para a quimioterapia, investigou a qualidade de vida e conforto do paciente através de oito aspectos: qualidade do sono, status psicológico, imagem pessoal, vestuário, complicações preocupantes, atividades da vida diária, vida social e atividades de trabalho. Em conclusão, os autores referem que o port-a-cath confere melhor padrão de uso comparado ao PICC ou ao convencional cateter central, pois o dispositivo implantando não impele a necessidade de mudança nas vestimentas, oferecendo risco mínimo de danos ou mal posicionamento pela ausência de uma linha externa.

Em desacordo, Bravo et al. ${ }^{12}$ afirmam que os pacientes que vivem com uma linha de PICC descrevem experiências positivas desde o momento de sua inserção, quando reconhecem tratar-se de um procedimento rápido e raramente doloroso, até a adaptação à vida com o cateter. Considerando os benefícios maiores do que os inconvenientes, o recomendariam a outros pacientes. 
É ponderoso salientar que esse estudo é composto por uma amostra de apenas 18 indivíduos e que embora a observação final tenha sido considerada otimista ao uso do PICC, algumas restrições nas atividades da vida diária foram registradas, a saber, os pacientes revelaram, por exemplo, desconforto relacionado à presença física do cateter, apresentando limitações à higiene, qualidade do sono, realização de atividades físicas, uso de roupas e até sobre a percepção positiva da própria aparência, pois o uso do dispositivo implicava na necessidade de um curativo no braço.

Tão importante quanto o bem-estar que proporciona, as implicações financeiras também foram consideradas quanto à escolha do dispositivo por parte do usuário. Tais custos, que se estendem desde o procedimento do cateterismo, manutenção, tratamento de complicações e remoção, determinam que muitos pacientes prefiram o PICC ao port-a-cath. Em análise isolada, o estudo de Fang et al..$^{25}$ sinaliza, ainda, que para tratamentos com duração inferior a 12 meses, o custo do port é muito maior que o do PICC. Entretanto, não há diferença significativa tratando-se de período superior.

\section{ventos adversos relacionados ao cateter}

Entre os inconvenientes precoces e tardios associados ao PICC, a trombose foi recorrente em todos os artigos que documentaram EA. Esse tipo de complicação, quando responsável pela remoção prévia do dispositivo, interrompe o curso pretendido do tratamento e tem potencial de causa para prejuízos financeiros e comprometimento do prognóstico da doença ${ }^{14}$.

A classificação de pacientes com câncer como integrantes do grupo de risco ao desenvolvimento de trombose já é bem documentada na literatura. Considera-se que a invasão do organismo hospedeiro pelas células neoplásicas provoca um desequilíbrio entre os fatores pró-coagulantes e anticoagulantes. Em um mecanismo complexo, essas células despertam a síntese de moléculas envolvidas no crescimento da massa tumoral, como o Fator Tecidual (FT), por exemplo, que suscita a produção de citocinas infamatórias, determinando maior expressão por parte do endotélio desse ativador primário da coagulação. Assim, a manifestação aumentada de FT, determinada direta ou indiretamente pelo quadro de neoplasia, efetiva os eventos trombóticos ${ }^{29}$.

Salienta-se que diferentes carcinomas exercem influências distintas sobre o desenvolvimento de TVP. Um estudo realizado na China, com o total de 8.028 pacientes recebendo tratamento antineoplásico por PICC para mais de 22 tipos de cânceres indica que, embora haja a necessidade de estudos mais aprofundados que fundamentem essa associação, o linfoma apresenta hegemonia em desenvolver episódios de trombose com quatro vezes mais chances sobre outros tipos de malignidades ${ }^{6}$.

A escolha do calibre ideal da veia de inserção consiste em manobra adequada à prevenção de eventos trombóticos. De acordo com a Infusion Nurses Society ${ }^{4}$, antes da inserção, considera-se adequado, por avaliação ultrassonográfica, o vaso cujo cateter corresponda a $45 \%$ ou menos do seu diâmetro, preservando o bom fluxo sanguíneo. Também, preconiza-se a seleção das veias basílicas, cefálicas, 
cubital mediana ou braquial, com predileção pela primeira por menor número de válvulas e maior lúmen ${ }^{5}$.

Sobre isso, Chen, Yu e $\mathrm{Li}^{14}$ reconhecem a importância de uma equipe de enfermagem qualificada em implantação do PICC, que além de conhecimentos teóricos, desenvolva habilidades técnicas a uma punção única e bem-sucedida, reduzindo lesões vasculares e consequente risco de TVP. Evidenciam, ainda, que mulheres com idade superior a 50 anos, pela redução do estrogênio, apresentam a viscosidade sanguínea aumentada e, portanto, devem ser enxergadas como potencial grupo de risco. Por fim, esse mesmo estudo considerou que o valor do Fibrinogênio (FIB) também se correlaciona diretamente à trombose venosa. A hipercoagulabilidade sanguínea, secundária à concentração de FIB plasmático, determina a necessidade de monitorização constante da função de coagulação do paciente, com o intuito de detecção e tratamento precoce de possíveis EA. Assim, o uso do PICC deve ser evitado ao máximo quando o FIB se apresentar maior que $4 \mathrm{~g} / \mathrm{L}$. Por outro lado, idade, lateralidade de membros, contagem de plaquetas, e Tempo de Protrombina não representaram ameaça à funcionalidade do dispositivo ${ }^{14}$.

Outra complicação dispendiosa, de consequências sistêmicas graves, como bacteremia ou sepse, com alta morbidade e mortalidade, compete à Infecção da Corrente Sanguínea Relacionada ao Cateter (ICSRC) $^{13,28}$. Como diagnóstico para esse tipo de infecção, utiliza-se o método da Diferença de Tempo de Positividade e a hemocultura pareada quantitativa. Para tanto, duas coletas sanguíneas de mesmo volume são realizadas com o intervalo máximo de 15 minutos, uma extraída do lúmen do cateter e outra de um sítio periférico. Com base na diferença de tempo entre hemoculturas positivas, interpreta-se como possível ICSRC quando a diferença entre o tempo de positividade de hemocultura central e periférica for maior que 120 minutos, notando que o microrganismo isolado deve ser o mesmo. Outros dois aspectos que podem ser observados para tal definição valem-se da identificação do mesmo microrganismo na ponta do cateter e na hemocultura periférica, bem como da presença de purulência no sítio de inserção coexistente à Infecção Primária da Corrente Sanguínea ${ }^{30}$.

Para a prevenção deste EA, a observação de três tipos de DAV inseridos em 335 pacientes com indicação de NPT, em regime domiciliar, realça o valor de instruções detalhadas ao paciente sobre a manutenção do cateter, além da necessidade de implementação de um rigoroso protocolo padronizado que inclui procedimento de lavagem asséptica do dispositivo, trocas semanais de curativos estéreis, e aplicação de conectores com válvula hemostática em todos os lúmens que não estejam em uso 28 .

Assim, Vashi et al. ${ }^{28}$, após depararem-se com uma baixa taxa de ICSRC, sugerem que sua incidência em pacientes imunocomprometidos, que recebem NPT, pode não estar relacionada ao tipo do 
dispositivo, mas ao protocolo de assistência usado, não evidenciando primazia entre ports, PICC ou cateteres centrais tunelizados para o desenvolvimento do quadro.

Não isento de limitações, releva-se que a origem dos artigos antepostos pode não traduzir a realidade nacional, considerando que a aplicabilidade de condutas clínicas relaciona-se com a estreiteza dos profissionais com o tema, o que, obviamente, varia de um lugar para outro. Além disso, a eleição única de publicações gratuitas pode ter negligenciado informações divergentes.

\section{CONCLUSÃO}

O resultado revelou que o PICC cresceu como tecnologia no tratamento de pacientes adultos e desempenha papel importante para a enfermagem oncológica na infusão a longo prazo de quimioterapia contra múltiplos tipos de cânceres, oferecendo acesso vascular confiável e conveniência ao usuário, especialmente por tratar-se de um cateter central cuja implantação não se configura como procedimento privativo de uma única categoria profissional, o que sustenta e simplifica sua escolha.

Entretanto, a perenidade do seu sucesso está inegavelmente vinculada à classificação adequada do paciente quanto à vulnerabilidade em desenvolver complicações trombóticas e infecciosas, bem como aos cuidados de enfermagem pós-inserção. Logo, eleva-se a importância de protocolos diligentes de manutenção e da educação contínua de clientes e acompanhantes sobre as práticas de autocuidado com o cateter.

Por essas complicações clínicas e por uma qualidade de vida associada a prejuízos na realização das atividades diárias e na percepção sobre a própria aparência, a depender da preferência pessoal de oncologistas e pacientes diante de outros dispositivos mais bem-sucedidos, o cenário futuro do PICC na terapêutica de pacientes adultos é incerto.

O Brasil carece de conteúdos dessa natureza e, julgando moderada a certeza de evidência, recomenda-se que estudos maiores sejam incentivados e desenvolvidos para avaliar o grau de satisfação do usuário e a opinião de especialistas sobre o tema.

\section{CONTRIBUIÇÕES}

Informa-se que todos os autores contribuíram igualmente na concepção do projeto de pesquisa, coleta, análise e discussão dos dados, bem como na redação e revisão crítica do conteúdo com contribuição intelectual e na aprovação da versão final do estudo.

\section{CONFLITO DE INTERESSES}

Nada a declarar. 
1. Alcantara DC, Peregrino AAF, Jesus CS, Siqueira AP, Silva PO, Marta CB, Silva RCL, Silva CRL. Cateter central de inserção periférica: contribuições para a enfermagem oncológica. Rev enferm UFPE on line [Internet]. 2019 [cited 2020 Mar 15]; 13(3): 715-731. DOI: 10.5205/1981-8963-v13i03a236058p715-731-2019

2. Fracchiolla NF, Todisco E, Bilancia A, Gandolfi S, Orofino N, Guidotti F, Mancini V, Marbello L, Assanelli A, Bernardi M, Santoro A, Cairoli R, Consonni D, Cortelezzi A. Clinical management of peripherally inserted central catheters compared to conventional central venous catheters in patients with hematological malignancies: A large multicenter study of the RELGROUP (Rete Ematologica Lombarda - Lombardy Hematologic Network, Italy). Am J Hematol [Internet]. 2017 [cited 2020 Mar 10]; 92(12):e656-659. DOI: 10.1002/ajh.24903

3. Campagna S, Gonella S, Berchialla P, Morano G, Rigo C, Zerla PA, Fuzzi R, Corona G, Storto S, Dimonte V, Mussa B. Can Peripherally Inserted Central Catheters Be Safely Placed in Patients with Cancer Receiving Chemotherapy? A Retrospective Study of Almost 400,000 Catheter-Days. Oncologist [Internet]. 2019 [cited 2020 Mar 15];24(9):e953-e959. DOI: 10.1634/theoncologist.2018-0281

4. Gorski L, Hadaway L, Hagle ME, McGoldrick M, Orr M, Doellman D. Infusion Therapy Standards of Practice. Infusion Nurses Society. J Infus Nurs [Internet] 2016 [cited Mar 13];39(1S):S1-S159. Available from: https://source.yiboshi.com/2017 0417/1492425631944540325.pdf

5. Pittiruti M, Scoppettuolo G. Gavecelt Manuale dei PICC e dei Midline. indicazioni, impianto, gestione. Roma (IT): Edra S.p.A.; 2017.

6. Zhang X, Huang J, Xia Y, Li C, Wang Y, Liu P, Bi X, Sun P, Lin T, Jiang W, Li Z. High risk of deep vein thrombosis associated with peripherally inserted central catheters in lymphoma. Oncotarget [Internet] 2016 [cited Mar 13]; 7(23):[about 7 p.]. DOI: https://doi.org/10.18632/oncotarget.8639

7. Shoutian L, Lv S, Liu Y, Wei G, Shi X, Chen S, Zhang X. The anticoagulants rivaroxaban and low molecular weight heparin prevent PICC-related upper extremity venous thrombosis in cancer patients. Medicine [internet] 2019 [cited 2020 Mar 10];98(47):e17894. DOI: 10.1097/MD.0000000000017894

8. Conselho Federal de Enfermagem (BR). Resolução - 258/2001: Inserção de cateter periférico central pelos enfermeiros [Internet]. São Paulo: COFEN; 2001 [cited 2020 Mar 12]. Available from: http://www.cofen.gov.br/resoluo-cofen-2582001 4296.html

9. Koo CM, Vissapragada R, Sharp R, Nguyen P, Ung T, Solanki C, Esterman A. ABO blood group related venous thrombosis risk in patients with peripherally inserted central catheters. $\mathrm{Br}$ J Radiol [Internet] 2018 [cited 2020 Mar 15];91(1082):20170560. DOI: https://doi.org/10.1259/bjr.20170560

10.Gudiol C, Nicolae S, Royo-Cebrecos C, Aguilar-Guisado M, Montero I, Martín-Gandul C, Perayre M, Berbel D, Encuentra M, Arnan M, Cisneros-Herreros JM, Carratalà J. Administration of taurolidine-citrate lock solution for prevention of central venous catheter infection in adult neutropenic haematological patients: a randomised, double-blinded, placebo-controlled trial 
(TAURCAT). Trials [Internet] 2018 [cited 2020 Fev 02];19(1):[about 1 p.]. DOI: https://doi.org/10.1186/s13063-018-2647-y

11.Louw V], Ntusi NAB. Virchow's triad revisited. S Afr Med ] [Internet] 2019 [cited 2020 Fev 12];109(11):[about 1 p.]. DOI: https://10.0.28.28/SAMJ.2019.v109i11.14442

12.Parás-Bravo $P$, Paz-Zulueta $M$, Santibañez $M$, Fernández-de-Las-Peñas $C$, Herrero-Montes $M$, Caso-Álvarez V, Palacios-Ceña D. Living with a peripherally inserted central catheter: the perspective of cancer outpatients-a qualitative study. Support Care Cancer [Internet] 2018 [cited 2020 Mar 18]; 26(2):[about 8 p.]. DOI: https://doi.org/10.1007/s00520-017-3815$\underline{4}$

13. He K, Wan Y, Xian S. Risk analysis on infection caused by peripherally inserted central catheter for bone tumor patients. J Cancer Res Ther [Internet] 2018 [cited $2020 \mathrm{Fev}$ 15];14(1):[about 3 p.]. Available from: http://www.cancerjournal.net/article.asp ?issn $=09731482$; year $=2018$; volume $=14 ;$ issue $=1 ;$ spage $=90$; epage $=93$; aulast $=$ He ;ype

14.Chen L, Yu C, Li J. Retrospective Analysis of Peripherally Inserted Central Catheterrelated Vein Thrombosis in Lung Cancer Patients. CJLC [Internet] 2015 [cited 2020 Fev 15];18(9):[about 4 p.]. DOI: https://doi.org/10.3779/j.issn.1009-3419.2015.09.04

15.Liang YJ, He Y, Li JM, Chen LM, Chen LP, Wang C, Ji L, Li ZX, Tang LQ, Chen QY, Fan YY, Hu $W$. The incidence and predictors of symptomatic venous thromboembolism associated with peripherally inserted central catheters in patients with nasopharyngeal carcinoma. Onco Targets Ther [Internet] 2018 [cited Fev 05];11:[about 8 p.]. DOI: https://doi.org/10.2147/OTT.S164723

16.Instituto Nacional de Câncer (BR). Ações de enfermagem para o controle do câncer: uma proposta de integração ensino-serviço. 3a ed. atual. amp. Rio de Janeiro: INCA; 2008.

17.Levasseur, N. et al. Optimizing vascular access for patients receiving intravenous systemic therapy for early-stage breast cancer-a survey of oncology nurses and physicians. Curr Oncol [Internet] 2018 [cited Fev 17]; 25(4):e298-e304. DOI: https://doi.org/10.3747/co.25.3903

18.Galvão CM, Sawada NO, Trevisan MA. Revisão sistemática: recurso que proporciona a incorporação das evidências na prática da Enfermagem. Rev Lat-Am Enferm [Internet] 2004 [cited Fev 05];12(3):[about 7 p.]. Available from: https://www.scielo.br/ pdf/rlae/v12n3/v12n3a14.pdf

19.Souza MT, Silva MD, Carvalho R. Revisão integrativa: o que é e como fazer. Einstein [Internet] 2010 [cited Fev 10];8(1):[about 4 p.]. Available from: https://www.scielo.br/ pdf/eins/v8n1/pt_1679-4508-eins-8-1-0102.pdf

20.Cecílio HPM, Oliveira DC. Modelos de revisão integrativa: discussão na pesquisa em Enfermagem. Atas - Investigação Qualitativa em Saúde [Internet] 2017 [cited Fev 10];2. Available from: https://proceedings.ciaiq.org/index.php/ciaiq2017/article/view/1272/1232

21. Whittemore $\mathrm{R}$, Knafl $\mathrm{K}$. The integrative review: updated methodology. J Adv Nurs [Internet] 2005 [cited Fev 25];52(5): [about 6 p.]. DOI: https://doi.org/10.1111/j.13652648.2005.03621.x 
22. Santos CMC, Pimenta CAM, Nobre MRC. A estratégia PICO para a construção da pergunta de pesquisa e busca de evidências. Rev Latino-Am Enferm [Internet] 2007 [cited Fev 15];15(3):[about 3 p.]. Available from: http://www.scielo.br/pdf/rlae/v15n3/ pt_v15n3a23.pdf

23.Galvão TF, Pansani TSA, Harrad D. Principais itens para relatar Revisões sistemáticas e Meta-análises: A recomendação PRISMA. Epidemiol Serv Saúde [Internet] 2015 [cited Fev 15];24(2):[about 7 p.]. DOI: https://doi.org/10.5123/S1679-49742015000200017

24.Ursi ES. Prevenção de lesões de pele no perioperatório: revisão integrativa da literatura [dissertação]. São Paulo: Universidade de São Paulo; 2005.

25. Fang S, Yang J, Song L, Jiang Y, Liu Y. Comparison of three types of central venous catheters in patients with malignant tumor receiving chemotherapy. Patient Prefer Adherence [Internet] 2017 [cited 2020 Fev 23];11:[about 7 p.]. DOI: https://doi.org/10.2147/PPA.S142556

26.Sá Neto JA, Silva ACSS, Vidal AR, Knupp VMM, Barcia LLC, Barreto ACM. Conhecimento de enfermeiros acerca do cateter central de inserção periférica: realidade local e desafios globais. Rev enferm UER] [Internet] 2018 [cited 2020 Mar 03];26:e33181. DOI: https://doi.org/10.12957/reuerj.2018.33181

27.Di Santo MK, Takemoto D, Nascimento RG, Nascimento AM, Siqueira E, Duarte CT, Jovino MAC, Kalil JA. Cateteres venosos centrais de inserção periférica: alternativa ou primeira escolha em acesso vascular? J vasc bras. [Internet]. 2017 June [cited 2020 Mar 07];16(2):[about 8 p.]. Available from: https://www.scielo.br/scielo.php? script $=$ sci arttext\&pid $=$ S167754492017000200104\&lng=en

28.Vashi PG, Virginkar N, Popiel B, Edwin P, Gupta D. Incidence of and factors associated with catheter-related bloodstream infection in patients with advanced solid tumors on home parenteral nutrition managed using a standardized catheter care protocol. BMC Infect Dis [Internet]. 2017 [cited 2020 Mar 10]; 17(1):[about 1 p.]. Available from: https://pubmed.ncbi.nlm.nih.gov/28558699/

29.Renni MJP, Cerqueira MH, Trugilho IA, Araujo Junior MLC, Marques MA, Koch HA. Mecanismos do tromboembolismo venoso no câncer: uma revisão da literatura. ] Vasc Bras [Internet] 2017 Out.-Dez. [cited 2020 Mar 03]; 16(4):[about 5 p.]. DOI: 10.1590/16775449.007817. Available from: https://www.scielo.br/pdf/jvb/v16n4/1677-5449-jvb-16-4308.pdf

30.Corrêa KLG. Almeida GMD, Almeida Junior JN, Rossi F. Diferença de tempo de positividade: método útil no diagnóstico de infecção de corrente sanguínea relacionada com cateter? J Bras Patol Med Lab [Internet] 2012 Jun [cited 2020 Mar 12]; 48(3):[about 7 p.]. Available from: https://www.scielo.br/pdf/jbpml/v48n3/a07v48n3.pdf 


\section{Correspondência}

René Rodrigues Pereira

E-mail: renemsccruises@yahoo.com.br

Submissão: $19 / 08 / 2020$

Aceito: 19/04/2021

Copyright@ 2021 Revista de Enfermagem UFPE on line/REUOL.

Este é um artigo de acesso aberto distribuído sob a Atribuição CC BY 4.0 Creative Commons Attribution-ShareAlike 4.0 International License, a qual permite que outros distribuam, remixem, adaptem e criem a partir do seu trabalho, mesmo para fins comerciais, desde que lhe atribuam o devido crédito pela criação original. É recomendada para maximizar a disseminação e uso dos materiais licenciados. 